\title{
On the Stability of Stellar Systems with Double Massive
} Centre

\author{
K.M. Bekarian \\ Department of Applied Mathematics, Yerevan State University, A. \\ Manugian 1, Yerevan, Armenia \\ A.A.Melkonian \\ Theoretical Department, Yerevan Physics Institute, Alikhanyan Br. str.2, \\ Yerevan, 375036, Armenia
}

\begin{abstract}
The Ricci curvature criterion is used for the investigation of the relative instability of several configurations of N-body gravitating systems. It is shown, that the systems with double massive centers are more unstable than the homogeneous systems and those with one massive center. In general this shows the efficiency of the Ricci curvature method introduced by Gurzadyan and Kocharyan (1987) for the study of N-body systems via relatively simple calculations, i.e. for small $N$, and hence small computer resources.
\end{abstract}

\section{Introduction}

The interest to the problem of dynamics of stellar systems with double massive centre is due to the discoveries of double nuclei in the centers of several galaxies have various separation in projection - from 2 pc for M31 up to 800 pc for Markarian 273; double massive object was descovered in the centre of Arp 220 [1] too. A massive binary system located in the core of a galaxy can lead to a number of dynamical effects.

In the present paper we investigate the role of double centers, i.e. binary massive bodies situated in the center of N-body systems, in the dynamical instability of the gravitating systems using the Ricci criterion (has been introduced in [2]) for estimation of the relative instability (chaos) of those systems. This criterion, for example, had enabled to establish in [3] that the regular central field is increasing the instability of the N-body systems. It is remarkable that this result has been obtained via numerical study of relatively small number of particles and later has been confirmed with extensive simulations on powerful computers 沟. The effect of the central regular field is crucial for the understanding of the relaxation, mixing and evolution of the galactic cores. The situation is complicated in a sense that there are both type of effects - acting to increase and decrease the chaos in the system. 
The flow of numerical methods range from the Lyapunov numbers and KS-entropy, approximate expansion and frequency maps up to powerful methods based on direct solutions [5], [6], [7]. The adequacy and efficiency of a given method in each particular case is itself an interesting problem. For example, the efficiency of estimation of Lyapunov numbers is limited by a number of reasons, particularly due to the exponential growth of the errors at large enough $\mathrm{N}$ inevitable at any iterated numerical procedure.

Geometrical methods based on the theorems of the theory of dynamical systems provide an alternative way of study of N-body systems via reducing the problem to that of the geometry of the phase and configurational spaces of the system [8]. In physical problems this method has been initially used by Krylov [9] and for N-body gravitational systems - by Gurzadyan and Savvidy [10]. In the latter papers it was shown that spherical N-body systems are K-systems, i.e. are mixing systems with exponential instability.

The statistical properies plays a key role for the understanding of the relaxation and evolution of many astrophysical objects - from the Solar system to galaxies and clusters of galaxies.

First, we will briefly describe the Ricci curvature formalism, and the algorithm of the numerical calculations.

\section{The Ricci curvature criterion}

N-body gravitating system with potential

$$
V=-G \sum_{i<j}^{N} G m_{i} m_{j} / r_{i j},
$$

$\left(r_{i j}\right.$ is the distance between the particles with masses $m_{i}$ and $m_{j}$ ) via a variational principle can be transformed to a geodesic flow in a Riemannian space [8]. The behavior of close geodesics in this space is described by the Jacobi equation

$$
\nabla_{u} \nabla_{u} n+\operatorname{Riem}(n, u) u=0,
$$

where $u$ is the velocity of geodesics, $n$ is the separation vector of close geodesics and $\nabla$ denotes the covariant derivative. For the normal component of the deviation one can obtain the following equation

$$
\frac{d^{2}\|n\|^{2}}{d s^{2}}=-2 K_{u, n}\|n\|^{2}+2\left\|\nabla_{u} n\right\|
$$


where

$$
K_{u, n}=\frac{[\operatorname{Riem}(n, u) u] n}{\|n\|}
$$

is the so-called two-dimensional curvature (Riem is the Riemannian curvature).

If $K_{u, n}$ is strongly negative in all two-directions $(\mathrm{u}, \mathrm{v})$ and everywhere in a compact manifold, the system possesses maximally strong instability properties is isomorphic to Bernoulli shift and is an Anosov system [11].

However this condition is too strong and therefore is not fulfilled for real physical systems. It is reasonable therefore to look for a weaker criterion using some average deviation of geodesics and a mean curvature in the manifold $M$. Consider the Ricci curvature

$$
r_{u}(s)=R_{\mu \nu} \frac{u^{\mu} u^{\nu}}{\|u\|^{2}},
$$

where $R_{\mu \nu}$ is the Ricci tensor.

The criterion of relative instability based on the Ricci curvature reads (Gurzadyan and Kocharian [2]):

The geodesics $\gamma_{1}(s)$ with velocity $u_{1}$ is more unstable with respect to the geodesics $\gamma_{2}(s)$ with velocity $u_{2}$ within some interval $\left[0 ; S_{1}\right]$, if

$$
r=\frac{1}{3 N} \inf \left[\mathrm{r}_{\mathrm{u}}(\mathrm{s})\right]
$$

and

$$
r_{1}<r_{2} ; r_{1}<0 \text {. }
$$

The advantage of this criterion is that it is checkable via computer simulations of many dimensional systems, including of N-body systems. Note also, that as distinct from the Lyapunov numbers, this criterion describes the local (in time) properties of the system.

\section{$3 \quad$ Numerical simulations}

At our computer experiments we estimated the Ricci curvature for several evolving configurations, i.e. traced the variation of the Ricci curvature in time. The formula for the Ricci curvature for N-body systems can be derived 
from the formulae given in previous section and has the following form [2], [12], [13].

$$
r_{u}(s)=-\frac{(3 N-2)}{2} \frac{W_{i k} u^{i} u^{k}}{W}+\frac{3}{4}(3 N-2) \frac{\left(W_{i} u^{i}\right)^{2}}{W^{2}}-\frac{(3 N-4)}{4} \frac{|\nabla W|^{2}}{W^{3}},
$$

where

$$
\begin{gathered}
W=E-V ; W_{i}=\frac{\partial W}{\partial q_{i}} \\
W_{i k}=\frac{\partial^{2} W}{\partial q_{i} \partial q_{k}} ; \Delta W=\sum_{i} W_{i i} ; \\
|\nabla W|^{2}=\sum_{i}\left(\frac{\partial W}{\partial q_{i}}\right)^{2} .
\end{gathered}
$$

We built the systems with $N=22$ using the scheme described in [2], i.e. we considered systems, so that the particles were located at the apexes of concentric cubes of unit sides. The velocities of particles were chosen in way to have no rotational momentum for the system.

We estimated the variation of the Ricci curvature by time for the following configurations (Figures 1-3):

1. Homogeneous, i.e. all particles have the same mass $m$;

2. With one central massive particle $m_{1}$, while $N-1$ particles have the same mass $m<<M$;

3. With two massive particles of masses $m_{1}$ and $m_{2}$ situated in the central part of the system, while $N-2$ particles have the same mass $m<<$ $m_{1}, m_{2}$;

Our calculations showed that the systems with massive center are more unstable than the homogeneous ones, thus confirming the conclusions in [2], [3], [4], [14]. Note, the growth of the instability with the increase of the central mass $M$.

Figures 1-3 illustrate the comparative instability of the three different types of systems mentioned above. The most unstable among the initial configurations is the system with double-central masses. Note, that as the systems evolves the Ricci curvature tends to zero for all three systems, however the rate of tending is most rapid for the system with double centers. Physically it is clear that the third type of systems has to dissolve quicker for small number of particles. Just this tendency has been also noticed at the numerical experiments, namely the tending to zero of the Ricci curvature becomes slower with the increase of $N$. 
In other words, the double massive central objects make the system more unstable initially, however then the system evolves quicker to its final dissolved state with regular orbits.

This tendencies have been confirmed in numerous experiments with configurations with various initial conditions.

\section{Conclusions}

Thus we used the Ricci curvature criterion to study the relative instability of three types of N-body systems: homogeneous systems, those with one central mass and two central massive bodies. The following main conclusions had been drawn via the numerical experiments:

1. The presence of the second massive central object makes the system more unstable as compared with that those of a single massive center and the homogeneous ones.

2. The system with double massive objects is evolving more quicker towards dissolution, i.e. to a more global regular situation.

3. The greater is the ratio of the mass of the massive objects to the mass of the rest particles, the more is the difference in the initial instability and the rate of evolution.

The main conclusion is however the efficiency of the Ricci curvature criterion for the study of such complex many dimensional systems - N-body systems - by means of simple numerical experiments.

We thank V.G.Gurzadyan and S.J.Aarseth for valuable discussions. This work is supported in part by INTAS grant.

\section{References}

[1] W. A. Baan, A. D. Haschil, ApJ, 454, 745, 1995.

[2] V. G. Gurzadyan and A. A. Kocharyan, Astrophys.Space Sci., 135, 307,1987 .

[3] V. G. Gurzadyan and A. A. Kocharyan, DAN SSSR, 301, 323, 1988.

[4] A. A. El Zant, V.G.Gurzadyan, Journ. Phys.D. 122, 241,1998.

[5] C. F. Gerald, P. O. Wheatley, Applied Numerical Analysis, (AddisonWesley Publishing Company, New York, 1994). 
[6] S. J. Aarseth, in Multiple Time Scales, ed. J. U. Brackbill and B. I. Cohen (Academic Press, New York, 1985); in Galactic dynamics and $N$-body simulations, ed. G. Contopoulos N. K. Spyrou and L. Vlahos (Springer-Verlag, New York, 1994).

[7] A. J. Lichtenberg, M. A. Lieberman, Regular and Stochastic Motion, (Springer Verlag, New York, 1983).

[8] V.I. Arnold, Mathematical Methods of Classical Mechanics, (Nauka, Moscow, 1979).

[9] N.S.Krylov, Papers on the Foundation of Statistical Physics, (AN SSSR Publ. (in Russian), Moscow-Leningrad, 1950).

[10] V. G. Gurzadyan and G. K. Savvidy, DAN SSSR, 277, 69, 1984; A \& A, 160, 203, 1986.

[11] D. V. Anosov, in Proceedings of the Steklov Institute of Mathematics, 90, ed. I. G. Petrovskii and S. M. Nikol'skii (American Mathematical society, Providence, Rhode Island, 1969)

[12] V. G. Gurzadyan A. A. Kocharyan Paradigms of the Large-Scale Universe (Gordon \& Breach, New York, 1994).

[13] V.G.Gurzadyan, D.Pfenniger Ergodic Concepts in Stellar Dynamics (New York: Springer-Verlag, 1994)

[14] A.A.El Zant, A \& A,326, 113, 1987. 


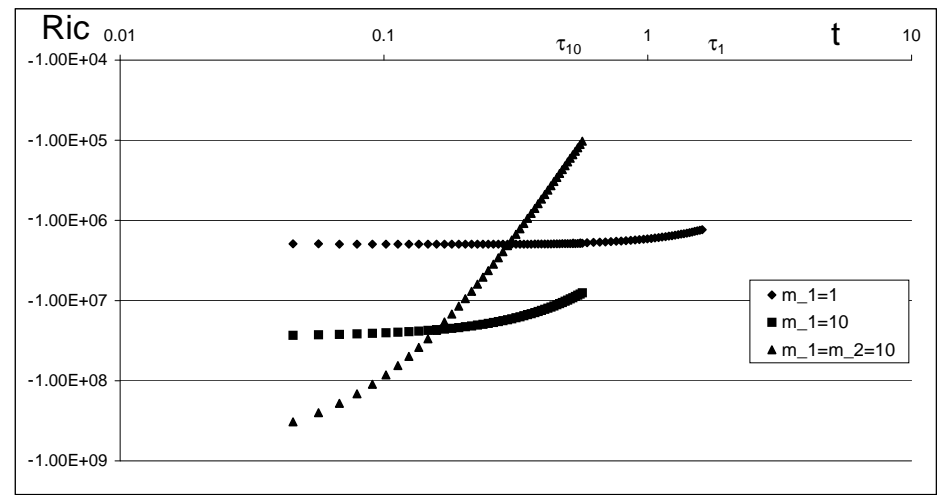

Figure 1: Comparative instability of three types of systems: with equal masses of particles, with a single central mass $m_{1}=10$ and with two massive centres $m_{1}=m_{2}=10$; here and in the following figures $m_{1}, m_{2}$ are the masses of the central particle/particles, while the other $N-1$ particles have $m=1$. 


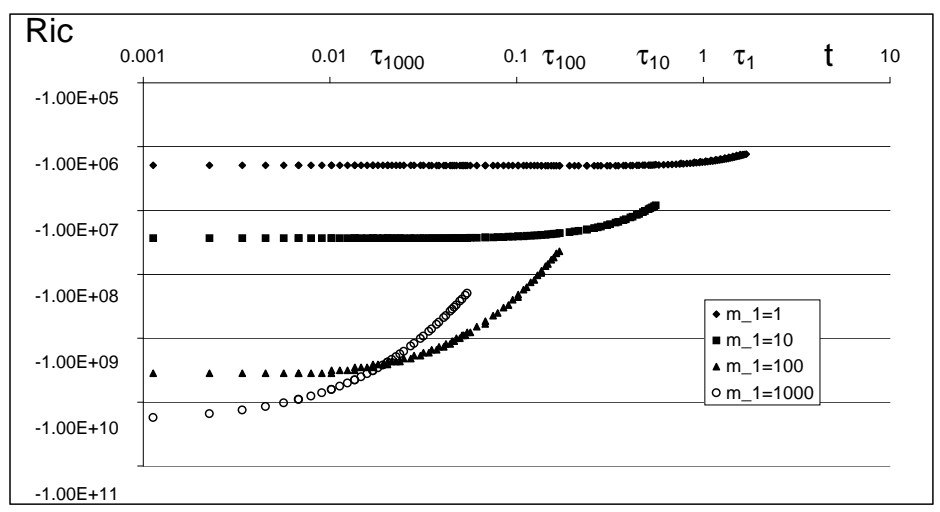

Figure 2: Comparison of configurations with various parameters of masses. 


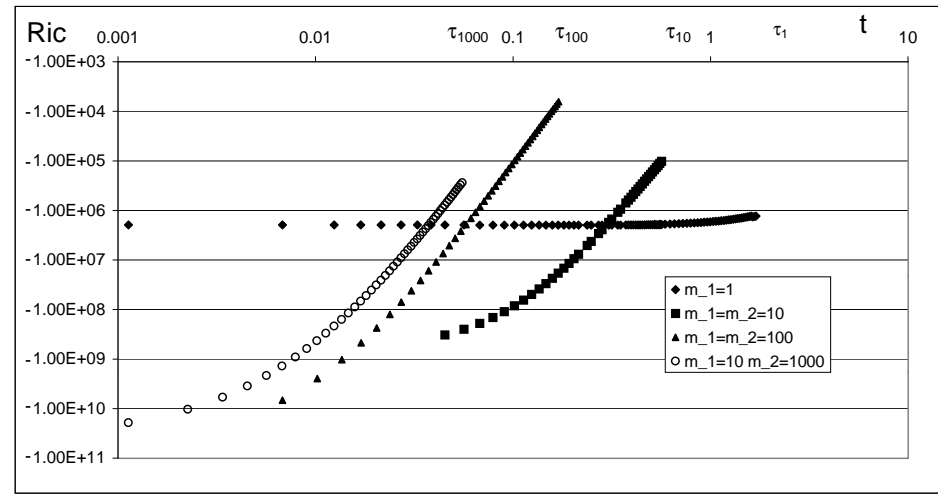

Figure 3: Comparative instability of systems with higher values of central masses. 